\title{
Análise cinemática da marcha em indivíduos com Acidente Vascular Encefálico
}

\author{
Gait cinematic analysis in subjects with cerebral Vascular Accident
}

Cristina Iwabe ${ }^{1}$, Maria Angélica da Rocha Diz ${ }^{2}$, Daniela Pinho Barudy ${ }^{3}$

\begin{abstract}
RESUMO
Introdução. A marcha torna o ser humano independente para suas atividades diárias. $\mathrm{O}$ déficit funcional ocasionado por lesões centrais, como o Acidente Vascular Encefálico (AVE) pode dificultar essa independência motora, principalmente na locomoção. Objetivo. analisar a cinemática da marcha em sujeitos com AVE. Método. foram incluídos 8 sujeitos com AVE, entre 61 a 76 anos de idade, sendo 4 hemiparéticos a direita e 4 a esquerda., analisando-se a variável espaço-temporal da marcha como: comprimento, duração e velocidade média da passada, utilizando-se o registro em vídeo e o software de imagem Dvideow 6.3 Barros. Resultados. Todos apresentaram alterações nos padrões cinemáticos da marcha, com menor duração e comprimento de passada, e maior velocidade do que os sujeitos normais. Conclusão. O comprometimento motor ocasionado pelas lesões do AVE produzem alterações nos padrões cinemáticos da marcha, para que haja a adaptação ás seqüelas neuro-sensoriais, as demandas da tarefa e do meio em que vivem.
\end{abstract}

Unitermos: Marcha. Acidente Gerebral Vascular. Paresia.

Citação: Iwabe C, Diz MAR, Barudy DP. Análise cinemática da marcha em indivíduos com Acidente Vascular Encefálico.

\section{SUMMARY}

Introduction. Gait becomes the individual independence for their daily activities. The functional deficit caused by central lesion as stroke, makes difficult this motor independence, mainly the locomotion. Objective. Analyze the kinematics gait in stroke patients. Method. It was included 8 patients with clinical diagnosis of stroke, 4 with hemiparesia on the right and 4 on the left. It was analyzed gait spatial-temporal parameters as: length, duration and average speed of the step, using the register in videotape and the software of image 6.3 Dvideow Barros. Results. All patients presented alterations in the kinematics standards of the gait, with lesser duration and length of step, and greater speed than normal subjects. Conclusion. The motor disorder caused by the central lesions produces alterations in the kinematics standards of the gait, in order to adapt the neuro-sensorial sequels, the demands of the task and the way where they live.

Keywords: Gait. Stroke. Paresis.

Citation: Iwabe C, Diz MAR, Barudy DP. Gait cinematic analysis in subjects with cerebral Vascular Accident.
Trabalho realizado no setor de Neurologia da Clínica de Fisioterapia do Centro Universitário Hermínio Ometto - Uniararas.

1. Fisioterapeuta - USP, Doutoranda em Ciências Médicas - Unicamp, Docente do Curso de Fisioterapia - Uniararas, Supervisora de Estágio em Neurologia - Unip Campinas.

2. Fisioterapeuta - Uniararas, Mestranda em Ciências da Motricidade Humana - UNESP Rio Claro.

3. Fisioterapeuta - Uniararas; Especialista em Fisioterapia Neurofuncional Adulto e Infantil - Uniararas.
Endereço para correspondência: Cristina Iwabe

Rua Mata dos Pinhais, 61/76 13082-761 Campinas, SP

E-mail: cristinaiwabe@uniararas.br / crisiwabe@hotmail.com

Recebido em: 29/07/08 Revisado em: 30/07/08 a 06/10/08

Aceito em: 07/10/08

Conflito de interesses: não 


\section{INTRODUÇÃO}

O acidente vascular encefálico (AVE) é caracterizado por início abrupto, de sintomas neurológicos focais ou globais, causados por isquemia ou hemorragia no cérebro ${ }^{1}$. Segundo a Organização Mundial da Saúde (OMS), o AVE é um sinal clínico de rápido desenvolvimento de perturbação focal da função cerebral, com mais de 24 horas de duração². As desordens cardíacas como a doença valvular cardíaca reumática, endocardite ou arritmias aumenta significativamente o risco de $\mathrm{um} \mathrm{AVE}^{3}$.

Durante os estágios iniciais é comum a flacidez sem movimentos voluntários. Progressivamente o quadro é substituído pelo desenvolvimento de padrões motores anormais decorrentes da alteração de tono muscular, perda de força muscular, reflexos posturais alterados e deformidades articulares ${ }^{3}$. Estas alterações músculo-esqueléticas frequentemente interferem nas habilidades motoras, essenciais para o desempenho de atividades e tarefas da rotina diária, como a marcha.

$\mathrm{Na}$ marcha normal a seqüência de movimentos depende do avanço sincronizado do corpo ao longo de uma linha de progressão. Isso depende da mobilidade articular e da ação muscular seletiva, para proporcionar a conservação da energia fisiológica $^{4}$. Nos sujeitos com AVE, aproximadamente 70\% retomam a sua capacidade de deambular ${ }^{5}$, embora sem o sinergismo muscular adequado. A marcha hemiplégica é caracterizada por alterações na fase de balanço e apoio devido principalmente a flexão dorsal do tornozelo e extensão do quadril insuficiente, impedindo o posicionamento adequado do pé e quadril ${ }^{6,7}$, alterando assim toda a dinâmica da marcha.

Muitos recursos para avaliação do padrão da marcha vêm sendo utilizados para se avaliar a biomecânica do movimento. A análise cinemática bidimensional vem sendo utilizada como uma importante metodologia de pesquisa e avaliação da marcha humana normal ou patológica, permitindo analisar as características espaço-temporais como comprimento, duração da passada e velocidade ${ }^{8,9}$.

As características espaço-temporais da marcha descrevem o aspecto quantitativo do padrão de movimento. A velocidade pode ser definida como a distância que o corpo percorre para frente na unidade de tempo; a duração da passada como o momento desde o contato inicial de um membro até o contato inicial seguinte do mesmo membro ${ }^{10}$; e o comprimento da passada é a distância (em centímetros) percorrido durante o tempo de dois toques sucessivos do mesmo pé ${ }^{11}$.
O objetivo deste estudo foi analisar as características cinemáticas da marcha em sujeitos com AVE, caracterizando e diferenciando os seus padrões espaço-temporais.

\section{MÉTODO}

\section{Amostra}

Foram incluídos 8 sujeitos com diagnóstico clínico de AVE, na faixa etária de 61 a 76 anos de idade, ambos os gêneros, sendo 4 com hemiparesia a esquerda e 4 com hemiparesia a direita, , apresentando marcha independente de auxílios externos, recrutados no setor de Neurologia da Clínica de Fisioterapia da Uniararas/Araras, assinando previamente o Termo de Consentimento Livre e Esclarecido, atendendo as normas do Comitê de Ética em Pesquisa (CEP) da Instituição. O estudo foi aprovado pelo CEP da Instituição, sob o parecer no 216/2006.

\section{Procedimento}

A coleta de dados foi realizada através da filmagem da marcha livre. Para registro dos dados cinemáticos foram utilizados marcadores circulares passivos posicionados na articulação do tornozelo (maléolo lateral), e pé (quinto metatarso e calcâneo) do membro parético. Os marcadores passivos eram esferas de isopor branco com aproximadamente $1,5 \mathrm{~cm}$ de diâmetro.

Os dados foram coletados em uma sala ampla com iluminação adequada. Uma faixa preta de 48 $\mathrm{cm}$ de largura foi colocada no solo, formando uma passarela de 3 metros de comprimento para limitar o espaço percorrido. Para a calibração do ambiente

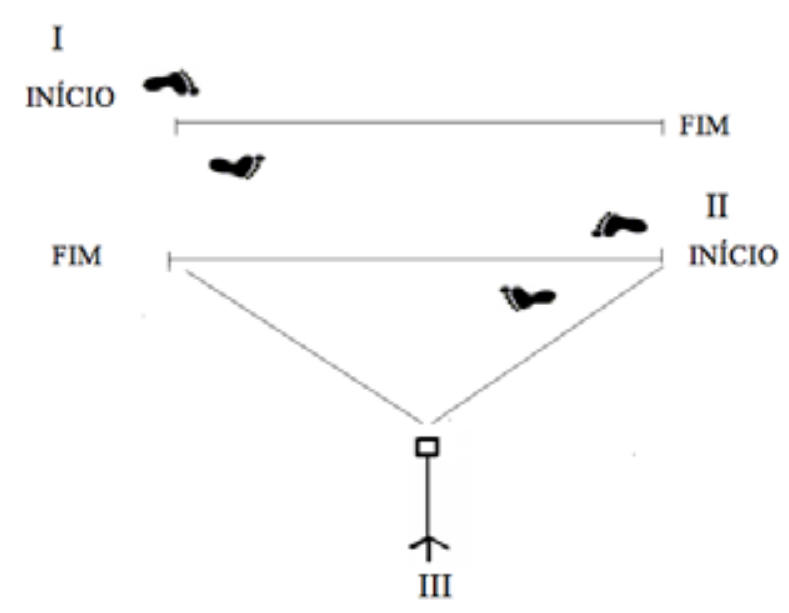

Figura 1. Representação esquemática do ambiente experimental para aquisição dos dados cinemáticos, indicando o início e o fim para pacientes hemiparéticos à direita (I), o início e o fim para pacientes hemiparéticos à esquerda (II) e posicionamento da câmera no plano sagital (III). 
experimental utilizou-se um calibrador em forma de cruz com $98 \mathrm{~cm}$ de altura e $93 \mathrm{~cm}$ de largura. Uma câmera (JVG modelo GR-AX650U), freqüência de $60 \mathrm{~Hz}$ foi posicionada sagitalmente a faixa preta, a uma distância de 7,14 m (Figura 1).

Durante o exame, os sujeitos vestiam shorts de cor escura, confortáveis, de forma que os movimentos das articulações não fossem restringidos durante a marcha livre. Antes de iniciar a coleta de dados, foram instruídos a andarem normalmente em uma velocidade preferida, percorrendo os $3 \mathrm{~m}$ de comprimento. Todos realizaram três tentativas, sendo a primeira considerada como familiarização do ambiente experimental (teste).

\section{Análise dos dados}

Para análise das filmagens foi usado vídeo cassete de quatro cabeças com reprodução de imagem quadro a quadro marca Panasonic e uma placa de vídeo 486DX-33, para capturar a imagem através do computador. Utilizando o software de imagem Dvideow 6.3 Barros ${ }^{11}$ foi possível obter as coordenadas $\mathrm{x}$ e y do tornozelo dos sujeitos nos instantes em que o pé tocou o solo. Com base nas coordenadas x e y foram possíveis calcular a duração e o comprimento da passada $(\mathrm{CP})$ do membro parético e a velocidade média, através de uma rotina realizada no software matemático Matlab ${ }^{12}$.

Uma passada foi definida como a distância entre dois eventos sucessivos do mesmo lado. Considerou-se a referência de análise, o membro hemiparético. A duração da passada (DP) foi obtida a partir da contagem do número de quadros do início ao término de cada passada. Esse número de quadros foi dividido pela freqüência de coleta de dados (60 $\mathrm{Hz}$ ) para ser convertida em segundos. A velocidade média (VM) de cada passada foi obtida pela divisão do CP pela DP. Os dados foram comparados grupo a grupo, de forma descritiva, comparando-os com dados na literatura.

\section{RESULTADOS}

Para todos as características analisadas foram feitas 2 tentativas, sendo que cada tentativa apresentou duas passadas. Para cada grupo de sujeito foram realizadas as médias da $1^{\circ}$ e $2^{\circ}$ passada, e a média total de cada tentativa.

$\mathrm{Na}$ análise do comprimento da passada (Tabela 1), observou-se que tanto o grupo hemiparéticos à esquerda (HE) quanto hemiparéticos à direita (HD) apresentaram CP semelhantes, aumentando o valor na segunda tentativa em ambos os grupos.
Tabela 1. Média dos valores do comprimento da passada $(\mathrm{cm})$ : grupo HE e HD.

\begin{tabular}{|c|c|c|c|c|c|c|c|}
\hline \multirow[b]{2}{*}{ Grupo } & \multicolumn{2}{|c|}{ Tentativa 1} & \multirow[b]{2}{*}{$\begin{array}{c}\text { Média } \\
\text { T1 }\end{array}$} & \multicolumn{2}{|c|}{ Tentativa 2} & \multirow[b]{2}{*}{$\begin{array}{c}\text { Média } \\
\text { T2 }\end{array}$} & \multirow{2}{*}{$\begin{array}{c}\text { Média } \\
\text { T1 e T2 }\end{array}$} \\
\hline & $1^{\mathrm{a}} \mathrm{P}$ & $2^{\mathrm{a}} \mathrm{P}$ & & $1^{\mathrm{a}} \mathrm{P}$ & $2^{\mathrm{a}} \mathrm{P}$ & & \\
\hline $\mathrm{HE}$ & 54.1 & 26.3 & 40.2 & 73.9 & 37.8 & 55.9 & 48.05 \\
\hline HD & 24.6 & 38.4 & 31.5 & 51.9 & 74.9 & 63.4 & 47.5 \\
\hline
\end{tabular}

$\mathrm{HE}=$ hemiparesia esquerda $\mathrm{HD}=$ hemiparesia direita $\mathrm{P}=$ passada $\mathrm{em} \mathrm{cm} ; \mathrm{T}=$ tempo de segundos

Na tabela 2, observa-se a duração da passada no qual os dois grupos apresentaram tempos semelhantes.

A análise da velocidade média demonstra que o grupo HE apresentou maior velocidade do que o grupo HD, nas duas tentativas propostas (Tabela 3).

\section{DISCUSSÃO}

O AVE acarreta seqüelas motoras e cognitivas importantes, que predispõe à instalação de deformidades, dor, insegurança e alteração do equilíbrio, dificultando a execução de uma capacidade funcional adequada. Este tipo de lesão no cérebro conduz a diminuição e/ou inervação inapropriada da musculatura voluntária afetando freqüentemente os músculos envolvidos na locomoção ${ }^{13}$. A marcha hemiparética é descrita como sendo lenta, laboriosa, abrupta devido aos variados graus de déficits na percepção, força, tono, controle motor, mobilidade articular e equilíbrio ${ }^{14}$.

Uma das características marcantes da marcha hemiparética é a assimetria do membro inferior afetado, percebido pela diferença do comprimento e duração da passada nas fases de apoio e de balanço, indicando comprometimento na capacidade de gerar e graduar as forças que controlam o movimento dos membros inferiores ${ }^{15}$. Essa assimetria, bem como as variações no comprimento e duração da passada foram observadas no nosso estudo, no qual apresentaram-se alteradas quando comparadas ao indivíduo normal, concordando assim com relatos prévios.

A velocidade média é automaticamente escolhida pelo indivíduo de acordo com a demanda da tarefa e do ambiente, de forma a gerar o menor gasto energético possível, com melhor equilíbrio funcional. Em indivíduos normais, a velocidade média da marcha é de aproximadamente 120 a $140 \mathrm{~cm} / \mathrm{seg}$, enquanto o comprimento da passada varia de 64 a $73 \mathrm{~cm}^{9}$.

Como podemos observar nos nossos resultados, os dois grupos apresentaram comprimento de passadas menores, e maiores velocidades médias do que o normal, principalmente no grupo HE. Isso resulta de estratégias motoras escolhidas para se adap- 
Tabela 2. Média dos valores da duração da passada (seg): grupo HE e HD.

\begin{tabular}{|l|c|c|c|c|c|c|c|}
\hline \multirow{2}{*}{ Grupo } & \multicolumn{2}{|c|}{ Tentativa 1 } & & \multicolumn{2}{|c|}{ Tentativa 2 } & & \multirow{2}{*}{ Média } \\
\cline { 2 - 7 } & $1^{\mathrm{a}} \mathrm{P}$ & $2^{\mathrm{a}} \mathrm{P}$ & $\begin{array}{c}\text { Média } \\
\mathrm{T} 1\end{array}$ & $1^{\mathrm{a}} \mathrm{P}$ & $2^{\mathrm{a}} \mathrm{P}$ & $\begin{array}{c}\text { Média } \\
\mathrm{T} 2\end{array}$ & T1 e T2 \\
\hline HE & 0.28 & 0.30 & 0.29 & 0.37 & 0.37 & 0.37 & 0.33 \\
\hline HD & 0.37 & 0.35 & 0.36 & 0.32 & 0.35 & 0.33 & 0.35 \\
\hline
\end{tabular}

$\mathrm{HE}=$ hemiparesia esquerda $; \mathrm{HD}=$ hemiparesia direita $; \mathrm{P}=$ passada $\mathrm{em} \mathrm{cm} ; \mathrm{T}=$ tempo de segundos.

tar as demandas da tarefa e do meio, associado ao comprometimento dos componentes neuromotores e músculo esqueléticos. Visto que o sujeito hemiparético não consegue realizar a transferência de peso adequada, devido aos déficits motores e/ou sensoriais, ele aumenta a velocidade e diminui o tempo em que o peso é transferido para o membro afetado, resultando em uma passada curta.

O padrão da marcha ceifante caracteriza-se pela diminuição da flexão e extensão dos membros inferiores, diminuindo a sua oscilação para frente e para trás, resultando em uma abdução exagerada do membro parético durante a fase de balanço ${ }^{16}$. A espasticidade nos flexores plantares pode ocasionar pé eqüinovaro ${ }^{17}$, e conseqüentemente dificuldade para flexão dorsal do tornozelo, comprometendo a absorção do impacto no contato inicial e hiperextensão do joelho durante toda fase de apoio. Subseqüentemente, as fases de apoio médio, apoio final e resposta à carga estarão comprometidos, diminuindo o comprimento do passo.

Em cerca de $70 \%$ dos hemiplégicos a alteração mais freqüente ocorre no nível da articulação do tornozelo, dificultando a flexão dorsal do pé durante a fase de balanço e apoio, impedindo o posicionamento adequado do pé18. Esta postura em eqüino do pé nas subfases de apoio final e pré-balanço acarreta perda da força propulsiva da marcha ${ }^{5}$, e conseqüentemente perda do mecanismo de redução do impacto no membro inferior e deslocamento do centro de massa no espaço durante a fase de resposta a carga. Todos esses mecanismos dificultam a redução do consumo energético na marcha ${ }^{6}$.

Não foi objeto de estudo a análise dos fatores neuromotores e músculo-esqueléticos que interferem no padrão de marcha em sujeitos com AVE, porém deve-se ressaltar que a avaliação propicia dessas características são fundamentais para a adequada conduta terapêutica. Considera-se que esses fatores primários (neuromotores e músculo-esqueléticos) podem ocasionar secundariamente alterações no padrão de marcha desses sujeitos.
O grupo HE apresentou maior velocidade da passada do que o grupo HD sugerindo que o grupo HE reduziu temporalmente as fases de apoio final e de balanço mais do que os do grupo de HD, como uma estratégia compensatória para continuar andando sem perder a estabilidade. Não há dados literários referentes a comparações na marcha entre os diferentes tipos de hemiparesia, sendo que a diferença encontrada no nosso estudo possa ser atribuída a extensão da lesão que pode ter sido maior nos sujeitos com déficits a esquerda, ocasionando assim maiores deficiências neuromotoras ou músculo-esqueléticas.

A fase de transferência de peso para o membro de suporte é essencial para a estabilidade do corpo e para que o próximo passo seja realizado. Neste caso quando o peso é transferido para o lado parético devido às alterações de tono, fraqueza muscular ou perda de mobilidade, a capacidade para manter apoio único do membro parético é diminuída e a estabilidade comprometida, resultando em uma marcha assimétrica sem acoplamento dos membros, com diminuição do passo.

Portanto, o tempo de apoio do membro parético é mais curto e a passada é menor do membro parético em relação ao membro não parético. Estes movimentos compensatórios necessários para a deambulação produzem um deslocamento anormal do centro de gravidade, resultando do gasto energético maior do que o normal ${ }^{19}$.

O fato da velocidade adotada pelos sujeitos do nosso estudo ter sido acima daquelas referidas pelos indivíduos normais ${ }^{19-21}$ e os comprimentos de passada serem menores, podem ser atribuídos ao aumento do tono muscular e fraqueza do membro acometido, obrigando-o a colocar rapidamente o membro não parético à frente, de modo a não perder o seu equilíbrio, e tentar restaurar o seu centro de gravidade para dar o próximo passo. A perda e recuperação do centro de gravidade fazem com que a marcha seja realizada de forma mais rápida para que ele não caia.

Tabela 3. Valores da velocidade média (cm/seg): grupo HE e HD.

\begin{tabular}{|l|c|c|c|c|c|c|c|}
\hline \multirow{2}{*}{ Grupo } & \multicolumn{2}{|c|}{ Tentativa 1} & & \multicolumn{2}{|c|}{ Tentativa 2} & & $\begin{array}{c}\text { Média } \\
\text { T1 e } \\
\text { T2 }\end{array}$ \\
\hline HE & 298.4 & 197.2 & 247.8 & 397.7 & 133.3 & 265.5 & 256.7 \\
\hline HD & 69.5 & 114.0 & 91.7 & 176.0 & 206.5 & 191.3 & 141.5 \\
\hline
\end{tabular}

$\mathrm{HE}=$ hemiparesia esquerda $\mathrm{HD}=$ hemiparesia direita $\mathrm{P}=$ passada $\mathrm{em} \mathrm{cm} ; \mathrm{T}=$ tempo de segundos. 


\section{CONCLUSÃO}

Sujeitos com AVE, tanto hemiparético a esquerda quanto a direita, podem apresentar alterações espaço temporais na marcha, com diminuição do comprimento e do tempo da passada, bem como o aumento da velocidade média, comparadas aos sujeitos sem alterações neurológicas. Tantos os sujeitos com hemiparesia direita quanto esquerda desse estudo apresentaram tais deficiências, resultando em conseqüente adaptação do mesmo, de acordo com as seqüelas neuro-sensoriais, às demandas da tarefa e do meio em que vivem.

De modo a não sobrecarregar o membro afetado, observou-se, portanto na nossa casuística, alteração nos padrões cinemáticos da marcha para tentar tornar essa capacidade funcional propicia para a sua independência. Deve-se salientar a necessidade de maiores números de sujeitos incluídos para que haja afirmação dos resultados observados.

\section{REFERÊNCIAS BIBLIOGRÁFICAS}

1. Lianza S. Medicina de Reabilitação. Rio de Janeiro: Guanabara Koogan, 2007, 474p.

2. Stokes M. Neurologia para Fisioterapeutas. São Paulo: Premier, 2000, pp. 83-99.

3. O'Sullivan SB, Schmitz TJ. Fisioterapia: Avaliação e Tratamento. 2a ed. São Paulo: Manole, 1993, pp. 385-426.

4. Tellini GG, Saad M. Análise da Marcha: Manual do Camo - SBMFR. São Paulo: Lemos Editorial, 1997, pp. 91-113.
5. Saad LMV, Castro LN. Análise da Marcha: Manual do Camo - SBMFR. São Paulo: Lemos Editorial, 1997, pp. 171-7.

6. Chin PL, Rosie A, Irving, M, Smith R. Studies in hemiplegic gait in advances in stroke therapy. In: Rose FC (ed.). Historical Aspects of the Neurosciences. New York: Raven Press, 1982, pp. 197-211.

7. Olney SJ, Richards C. Hemiparetic gait following stroke. Part 1: characteristics. Gait Post 1996;4:136-48.

8. Araújo AGN, Andrade LM, Barros RML. Sistema para análise cinemática da marcha humana baseado em videogrametria. Fisioter Pes 2005; 11(1):3-10.

9. Melanda A. Fisioterapia Aspectos Clínicos e práticos da Reabilitação. São Paulo: Artes Médicas, 2005, pp. 615-42.

10. Kerrigan DC, Schaufele M, Wen M. Análise da marcha. In: Delisa JA. Tratado de Medicina e reabilitação. $3^{\text {a }}$ ed. São Paulo: Manole, 2002, pp. 177-98.

11. Sutherland DH, Kaufman KR, Moitoza JR. Cinemática da marcha humana normal. In: Rose J, Gamble JG. Marcha Humana, 2a ed. São Paulo: Premier, 1998, pp. 23-44.

12. Barros RML, Brenzikofer R, Leite NJ, Figueroa PJ. Desenvolvimento e avaliação de um sistema para análise cinemática tridimensional de movimentos humano. Rev Bras Eng Biomed 1999;15(12):79-86.

13. Hanselman D, Littlefield B. Matlab 6: curso completo. São Paulo: Pearson, 2003, 676p.

14. Esquenazi A, Hirai B. Gait analysis in stroke and head injury. In: Graik RL, Oatis A. Gait analysis: theory and application. St. Louis: Mosby-Year Book, 1995, pp. 412-20.

15. Shumway-Cook A, Woollacott MT. Controle Motor. Teoria e Aplicações. São Paulo: Manole, 2003, pp. 289-380.

16. Rowland LP. Merrit Tratado de neurologia. $9^{\text {a }}$ ed. Rio de Janeiro: Guanabara Koogan, 1997, 1188p.

17. Edwards S. Fisioterapia neurológica: uma abordagem centrada na resolução de problemas. Porto Alegre: Artmed, 1999, 224p.

18. Yavuzer MG. Walking after stroke: Interventions to restore normal gait pattern. Ankara: Pelikan Publications, 2006, 136p.

19. Lucarelli PRG, Greve JMA. An Alteration of the load-response mechanism of the knee joint during hemiparetic gait following stroke analyzed by 3-dimensional kinematic. Clinics 2006;61(4):295-300.

20. Perry J. Análise da Marcha. Barueri: Manole, 2005, pp. 125-7.

21. Barela JA. An examination of constraints affecting the intralimb coordination of hemiparetic gait. Hum Mov Sci 2000;19:251-73. 